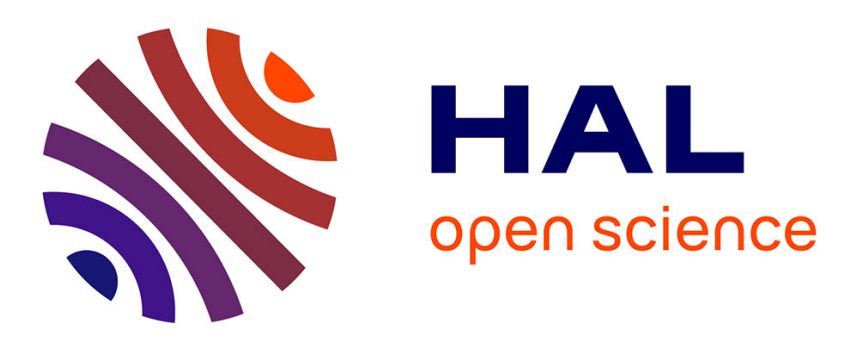

\title{
Using protein-ligand docking to assess the chemical tractability of inhibiting a protein target
}

\author{
Richard A. Ward
}

\section{To cite this version:}

Richard A. Ward. Using protein-ligand docking to assess the chemical tractability of inhibiting a protein target. Journal of Molecular Modeling, 2010, 16 (12), pp.1833-1843. 10.1007/s00894-0100683-y . hal-00575683

\section{HAL Id: hal-00575683 \\ https://hal.science/hal-00575683}

Submitted on 11 Mar 2011

HAL is a multi-disciplinary open access archive for the deposit and dissemination of scientific research documents, whether they are published or not. The documents may come from teaching and research institutions in France or abroad, or from public or private research centers.
L'archive ouverte pluridisciplinaire HAL, est destinée au dépôt et à la diffusion de documents scientifiques de niveau recherche, publiés ou non, émanant des établissements d'enseignement et de recherche français ou étrangers, des laboratoires publics ou privés. 


\section{Editorial Manager(tm) for Journal of Molecular Modeling Manuscript Draft}

Manuscript Number: JMM01093R1

Title: Using Protein-Ligand Docking To Assess The Chemical Tractability Of Inhibiting A Protein Target

Article Type: Original paper

Keywords: Protein-Ligand Docking; Druggability; Ligandability

Corresponding Author: Dr. Richard A Ward,

Corresponding Author's Institution: AstraZeneca

First Author: Richard A Ward

Order of Authors: Richard A Ward

Abstract: Assessing the difficulty of inhibiting a specific protein by a small molecule can be highly valuable in risk-assessment and prioritisation of a new target. In particular, when the disease linkage for a number of targets is broadly similar, being able to identify the most tractable can have a significant impact on informing target selection. With an increasing focus against new and novel protein classes, being able to assess the most likely targets to yield lead-like chemical start points can guide the selection and the lead-generation strategy implemented. This study exploits protein-ligand docking studies on published protein $x$-ray crystal structures to provide guidance on the feasibility of identifying small molecule inhibitors against a range of targets.

Response to Reviewers: 1) I am not convinced that a biased fragment library (the bias is introduced via the filter criteria for $\log \mathrm{P}, \mathrm{MW}$, and other parameters) should be able to judge the tractability of any target in general. What if potent fragment binders could not idenitified via docking just because they were removed from the initial fragment collection because of the filter criteria ? This concern is somehow reflected already in Fig 7A and 7B. A more general characterization of the fragment library (as expressed by the mean score of the top 10\% docking hits) is only hardly able to distinguish between druggable and "prodrug" binding sites (SP) or "prodrug" and undruggable sites (XP) . A more rigorous approach could for instance be based on a random selection of compounds rather than on a biased fragment library.

The key criteria used to build this fragment library is chemical/structural diversity (there is a reference to the details behind how this library was built). This ensures that the library covers a broad range of scaffolds and interaction types. This allows the mapping of a target binding site by fragment screening (experimentally or computationally). The reason for focusing this fragment library around the stated criteria is that fragment hits with properties in these ranges are suggested as being good chemical start points for fragment-based lead generation projects where achieving an orally bioavailable drug is the aim (i.e. a molecule largely compliant with Lipinski-like criteria). See the report below (also reference 12) from Astex on 'Rule of Three' criteria. Our fragment library, however, has not been filtered as harshly as this library as we want to maximise the information from the screen (which is to some extent addressing the issue raised by the reviewer).

12. Congreve M, Carr R, Murray C, Jhoti H A (2008) 'Rule of Three' for fragment-based lead discovery? Drug Discovery Today 8:Issue 19:876-877 
The other key reason for using this library is that published fragment libraries (filtered similarly to the library I have used in this study) have been used to assess drugability using experimental screening approaches as shown in the reference 4 in the manuscript (below). Therefore there is validation for the use of libraries filtered by these criteria to assess drugability experimentally so I believe it is a suitable fragment set to assess targets computationally. Due to molecule complexity arguments (Andrew Leach's work for example) then you can sample chemical space well at the fragment level but when the compounds get larger then the coverage of chemical space is much smaller, this is the main reason why MW cut-offs have been used. So an aim of my work is to try and get similar information from this computational approach as what you get experimentally, which does mean using similar fragment sets.

\section{Hajduk PJ, Huth JR, Tse C (2005) Predicting protein drugability Drug Discovery Today 10:23- $24: 1675-1682$}

It is true that some targets may produce tighter binding hits with fragments outside of these criteria but these are going to be more challenging to develop into a lead series (and then a drug candidate). For example if fragments identified are more lipophillic (i.e. ClogP $>3 / 4$ ) then whilst the fragments are developed into a lead series the lipophillicity will further increase and it will be difficult to achieve a drug with suitable properties to show oral bioavailability. And larger fragments will not cover chemical space as efficiently. So identifying tight binding fragments with unwanted properties may not be a useful indication of drugability, although clearly this information may tell you something about what the protein 'wants'. However, as oral bioavailability may not always be the aim I agree that it would be interesting to consider fragment sets with higher and lower lipophillicity for example. This will be particularly useful for considering targets such as protein-protein interactions, and due to the possible limitations of using screening techniques on more lipophillic (and likely less soluble fragments) then it could be particularly powerful. This is beyond the scope of the work in the manuscript but something I will have a look at. I have made some modifications to the manuscript to address this comment.

I believe the reasoning that using the top $10 \%$ of hits is less successful is mainly down to the differences in the profile of the docking scores across the fragment set across different targets (i.e. some have quite steep profiles, some flatter). This is also the reason for looking at the data in the form of figure 8 for an alternative view. Therefore using a rigid rule like the top $10 \%$ wasn't successful, along with other measures assessed. The mean score remains as being the most useful in capturing the information in the docking scores and giving good separation for the 'druggable' and 'undruggable' categories, which is clearly the major aim. If a random subset of the fragment set was used instead then the conclusion would be similar to the full set in most cases. However it is important to ensure that the fragment set is a sufficient size to contain good diversity and in terms of covering chemical space.

2) Secondly, even if a high (in-silico) affinity binder is found, it is not guaranteed that this fragment can be grown into a potent and drug-like lead. What is the link between an in-silico fragment affinity (docking score) and the final profile of an inhibitor? This is of special relevance for the class of 'prodrugs / transporters'. The active principle of prodrugs very often reaches high potencies on the target level. Why should fragments bind less tight to these targets?

There is significant literature around (and linked above) that fragments with properties in the 'Rule of 3 '-like space are suitable start points for the delivery of an orally bioavailable drug. A number of the references in the manuscript address this. The ability to identify tight binding fragments for a target doesn't always mean that they can be elaborated into a candidate drug (that's the reasoning for differentiating the terms 'drugability' and 'ligandability') but the ability to find fragment hits against a target is an important first step in understanding if finding high affinity ligands may be possible. So its shown that if you can't find fragment hits then it will be very difficult to identify a candidate drug. This information helps with the risk assessment against a target. I agree that the 'prodrug/transporter' 
issue is less clear, this is why they have been separated in the Cheng paper and why I also kept them separate. You can clearly identify potent inhibitors against these targets, but in most cases the key functionality has to be masked due to the warhead being highly charged (or reactive) for example. So the reason why the fragments tend to bind less well to these targets is likely due to the fact that these highly charged warheads are not present in the fragment set but are required for tight binding. However simple acids, bases and charged groups are populated in the fragment set I have used. I think an alternative fragment set may be required to fully assess these targets but the fragment set I've used is still answering the key question around conventional drugability (i.e. likelihood of identifying an orally bioavailable drug against the target). I have added some text around this in the revised report, this also links in with the comments below around correlations with $\mathrm{MW} / \mathrm{ClogP}$. I have also added this reference into the manuscript.

30. Rautio J, Kumpulainen H, Heimbach T, Oliyai R, Oh D, Järvinen T, Savolainen J (2008) Prodrugs: design and clinical applications. Nature Reviews Drug Discovery 7:255-270

3) Is there any reason to skip certain targets from the Cheng study ? For a proper head-to-head comparison (which is the key point in this manuscript) the entire data set should have been investigated. In general, a statistical assessment (like for instance p values for the separation of two classes) would be important.

I have added the $\mathrm{p}$-values for the key results in Figure 6 (p-Value $=0.0052$ for SP protocol and $\mathrm{p}$ Value $=0.0041$ for XP) and Figure 7 ( $p$-Value $=0.0212$ for SP protocol and $p$-Value $=0.0944$ for XP) . I analysed what I considered to be a reasonable number of the Cheng targets to satisfy myself that the results were largely consistent with their approach and the assessment of the target from the known inhibitors. I covered the majority of the 'undruggable' and 'transporter/prodrug' targets (which had the smallest number of targets) and sampled from the larger 'druggable' set. I did not miss any results out of my manuscript I just chose not to run them all due to other time pressures at work. In the manuscript I have looked in more detail at specific targets and in some cases considered a greater number of protein structures in the drugability assessment than in the Cheng paper. I therefore chose to look in more detail at a smaller numbers of targets, but enough to be able to perform a comparison. I am now running the approach on novel, in-house targets which I hope to publish at a later date although these are confidential at present. I have made some modifications to the manuscript to explain this further.

4) What is the conclusion from the differences between XP and SP ? If computational speed is the only factor to differentiate between both protocols, one of them can be skipped. I would then suggest to take a completely different docking protocol into account as second approach.

Going forwards I would use the SP protocol routinely. I have made this more obvious in the manuscript.

5) Is there a correlation between MW or logP and the docking score for any particular target ? Even if this is not the case - were ligand efficiencies considered as criterion to distinguish druggable from nondruggable sites?

I had looked at this, none of the targets showed any convincing trend between MW and ClogP. I had reported the mean scored across all targets (Figure 2) to try and show this. Interestingly, any targets tested in future which do show a significant correlation with MW or ClogP may suggest that fragments with higher affinity may be found outside this property space, linking in with some of the comments above. I have added some additional comments around this in the manuscript. I did also looked at ligand efficiencies (docking score/heavy atoms) but the separation between the 'drugable' and 'undrugable' categories using this measure was made worse. Plot shown below for SP protocol data. 
SEE ATTACHED FILE FOR GRAPH.

6) How do the docking scores (say for the top fragment) compare to the hightest experimental affinity that is known for each target? This would be more meaningful than a comparison with the Cheng model.

The issue I have with this method is that it uses the docking score of a single fragment against a target to represent the ligandability for that target. Docking scores are not robust or accurate enough to allow the score for a single fragment to be able to represent a target. This is why a diverse fragment set was docked and the mean score used to allow differentiation of targets. The plots below show the results of doing this analysis of the top fragment against the highest experimental efficiency, clearly there is no correlation. I also believe that the specific choice of fragments screened would then be more important for this method to work, which was one of the concerns raised in the first point.

\section{SEE ATTACHED FILE FOR GRAPH.}

Additionally, reflecting how experimental druggability data is used to assess a target although the most potent fragment identified is of interest the hit rate and numbers of fragments with measurable binding is also key to fully assess drugability. Therefore the protocol I have used of considering the mean fragment score from the docking to represent docking is probably the most comparable to this, along with giving the best separation between 'drugable' and 'undrugable' categories. 
Graphical abstract
Click here to download Abstract graphic: Graphical Abstract.doc
Graphical Abstract

Graphical abstract
Click here to download Abstract graphic: Graphical Abstract.doc
Graphical Abstract

Graphical abstract
Click here to download Abstract graphic: C

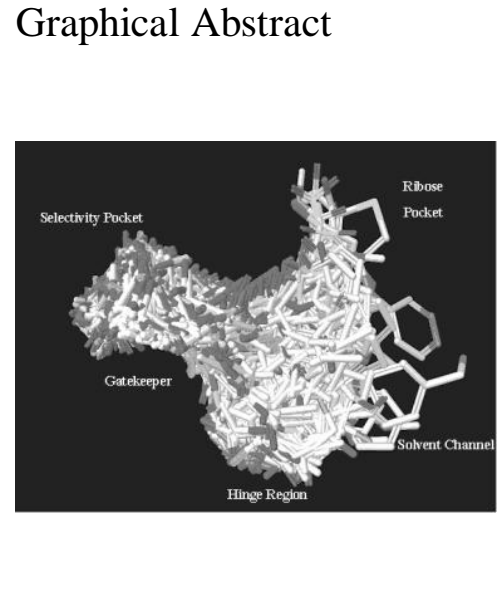

$(2+2$

(2)

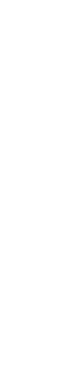

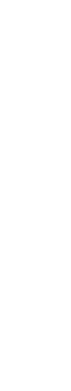




\title{
Using protein-ligand docking to assess the chemical tractability of inhibiting a protein target
}

Received: 09.11.2009 / Accepted: 09.02.2010

Richard. A. Ward ${ }^{\bowtie}$

Cancer \& Infection Discovery, AstraZeneca, Alderley Park, Macclesfield, SK10 4TG

${ }^{\square}$ Phone: +44 (01625) 519045; Email: richard.a.ward@astrazeneca.com

\begin{abstract}
Assessing the difficulty of inhibiting a specific protein by a small molecule can be highly valuable in risk-assessment and prioritization of a new target. In particular, when the disease linkage for a number of targets is broadly similar, being able to identify the most tractable can have a significant impact on informing target selection. With an increasing focus against new and novel protein classes, being able to assess the most likely targets to yield lead-like chemical start points can guide the selection and the lead-generation strategy implemented. This study exploits protein-ligand docking studies on published protein x-ray crystal structures to provide guidance on the feasibility of identifying small molecule inhibitors against a range of targets.
\end{abstract}

Keywords Protein-ligand docking $\cdot$ Druggability $\cdot$ Ligandability 


\section{Introduction}

It has been reported that $60 \%$ of small-molecule drug discovery projects fail due to the lack of suitable leads [1]. The term 'druggability' has subsequently been used to capture the feasibility of inhibiting a specific target with an orally bioavailable small molecule [2]. Some estimates indicate that only $10 \%$ of genes in the human genome are 'druggable' and only $5 \%$ are druggable and relevant to disease [3]. The druggability of a target is currently assessed in a number of ways, this includes studying known inhibitors from the gene family and fragment/subset screening [4]. Fragment and subset screening have be deployed against targets to identify the ease of finding small molecule binders which can then indicate the likelihood of success by HTS and or random screening. The data from these studies is encouraging although some targets may not be suitable for such screening campaigns and also significant resource is required to carry out this work on a set of proposed targets in a timely fashion. Analyses of a target binding site can also be exploited where suitable crystal structures are available [5, 6]. Such analyses can utilize information on the size, concavity and electrostatic nature of a pocket; one such recent report translates this information into a Maximal Affinity Model [7]. This study highlights how a target with a well-defined pocket with a suitable balance of properties will score more highly than an open and feature-less pocket which can be observed in some protein-protein interactions. This approach is restricted to targets which have suitable protein crystal structures but encouragingly new and novel $\mathrm{x}$ ray crystal structures continue to be published [8]. Protein movement and flexibility is also an issue as some pockets are only fully formed when a ligand is bound [9]. Finally, analysis of the protein family or class can be used to estimate druggability. So for example if a proposed novel target is a kinase and a related family member has been shown to be tractable you might assume the druggability is likely to be comparable [10]. Clearly if a novel protein family or target class is being assessed there may be little information in the literature to provide this guidance.

The study detailed in this paper uses a test-set of 950 diverse fragment-sized molecules [11] to allow an in silico druggability prediction through docking against a target binding site. This can be considered as the computational equivalent of a fragment screen, but where a specific pocket is targeted by each run. These fragments have been filtered using previously reported criteria and largely consistent with reported fragment library characteristics [11, 12]. 
Although docking scores do not consistently show a correlation with ligand binding affinities [13], the expectation is that a druggable binding site is likely to score fragments more highly when productive hydrogen bonding and lipophillic interactions with the protein can be identified. This measure of assessing the druggability of a binding site might be more accurately described as 'ligandability', where a protein of high ligandability is likely to bind fragments with a reasonable affinity. Identifying ligands which bind to a protein may not necessarily translate into finding a drug if the nature of the binding site does not allow the affinity of the lead to be increased whilst maintaining the required drug-like properties [14]. However, an indication that fragments can be identified for a target protein gives some confidence that the target might be tractable and or a tool compound may be identified for target validation work. Additionally, if this information could be obtained computationally it would allow a greater number of targets to be considered than using experimental approaches.

\section{Materials and methods}

\section{Fragment set}

A set of 950 diverse fragments were identified for this study. The distributions of ClogP (Version 4.3), molecular weight and rotatable bonds are shown for this fragment set below in Fig. 1.

$<$ Figure 1>

The fragment set has a mean $C \log P$ of 1.6 and the mean molecular weight was 188 . The number of rotatable bonds in the set is centered on two, with a range between 0 and 6 . The number of hydrogen bond acceptors and donors were also kept relatively low. Compounds with functionality not compatible with good fragment leads were also removed from the set. The reasoning behind using a fragment set filtered to these criteria was to provide consistency to the druggability assessments which have been run using experimental approaches on similarly filtered fragment libraries [4]. 


\section{Protein preparation}

The publicly available protein structures used in this study were taken from the RSCB Protein Data Bank [15]. The proteins were prepared for docking and analysis using the Protein Preparation Wizard in Maestro [16]. Hydrogens were added, reflecting the likely protonation states of amino acids at $\mathrm{pH}=7.4$. The hydrogen bonding environments of amino acids such as Asn and His were analyzed to ensure that the most likely conformation had been assigned [17]. Unless an interface between dimer/tetramer units was required for binding a single monomer unit was used for the docking. Where a suitable ligand/co-factor was present in the pocket, it was used to define the binding site for the docking. Where no ligand was present, SiteMap [18] was used to identifying probable binding sites, the primary pocket was then manually defined using amino acid residues.

\section{Protein-ligand docking}

The likely protonation and tautomeric states of the 950 compound set was enumerated in Leatherface [19]. This produced around 1150 distinct chemical structures. A 3D-structure of each molecule was produced in CORINA [20], which was then optimized using the MMFF94 Forcefield [21], and the Sheffield solvent model [22]. These structures were then used as input into the docking calculations. Glide was used for the protein-ligand docking [23]. Standard precision (SP) and extra precision (XP) docking protocols were run for comparison. The highest scoring pose for each compound (which includes the various protonation/tautomeric states) was used to represent the score for that compound against the target.

\section{Protein structures}

The following tables show the X-ray crystal structures used for the docking studies. PDB codes of proteins from the RCSB protein data bank are provided [15].

$<$ Table 1> 


\section{Results and discussion}

\section{Correlations of docking score against physiochemical properties}

It has been demonstrated that docking scores can correlate with the molecular size of the compounds docked [13]. To ensure that this was not the case in this study the mean docking score of each fragment was calculated across all the protein targets studied. This value was then plotted against molecular weight and $\mathrm{ClogP}$ from the Standard (SP) and Extra Precision (XP) docking protocols. Fig. 2 shows that there is no significant correlation with the molecular weight or ClogP of the fragment and the mean docking score across the targets.

$<$ Figure 2>

Interestingly, the XP protocol shows a stronger trend, albeit still a weak one, between the mean docking score of a fragment across the targets and Molecular Weight/ClogP (Fig. 2D). The RSquare $=0.22$ for XP plotted against Molecular weight in Fig. 2D can be compared with an RSquare $=0.08$ with SP in Fig. 2B. The correlation of the docking scores against MW and ClogP was also examined for individual targets but there were no clear trends. Any assessed targets, which do show a significant correlation, may indicate that higher affinity fragments could be identified outside the property ranges in the fragment set.

\section{Comparison work with maximal affinity prediction model (MAPPOD)}

This section of the study compared a number of the systems published in the Maximal Affinity Model (MAP $\left.{ }_{\mathrm{POD}}\right)$ method to provide an assessment of how the two approaches compare with each other. The majority of studied targets classified as 'undruggable' and 'prodrug/transporter' were analyzed as these contained relatively small numbers of enzymes. The 'druggable' targets were then sampled from to enable a reasonable assessment against this approach. For a number of these targets additional protein structures have been considered (see Table 1) than in the approach reported by Cheng et al. [7] to maximize the available binding site information. For this assessment, the mean docking score of all 950 fragments docked to the target protein was used as an indicator of ligandability. Where multiple protein structures were used, the mean docking score across all the structures was 
taken. Note that a more negative docking score shows an improvement in the predicted affinity of the fragments. To assess how the ligandability prediction from the SP and XP docking protocols compare, they are plotted against each other in Fig. 3.

$<$ Figure 3>

The analysis demonstrates that the SP and XP protocols correlate reasonably well (RSquare $=0.89$ ) and as XP is $\sim 10 \mathrm{x}$ slower than SP it may not add additional significant value. However, it may be prudent to run both protocols as some targets may vary in their predictions and as Glide can be run using parallel processing the additional computational resource is small. Additionally, as these docking algorithms develop it is a useful crosscheck to ensure that they give a consistent view with previous observations. The SP and XP protocols were then compared to the published $\mathrm{MAP}_{\mathrm{POD}}$ [8] for a subset of the previously published targets (Table 1), the MAP $\mathrm{P}_{\mathrm{POD}}$ was converted into a pKd for this comparison (Fig. $4)$.

$<$ Figure 4>

The comparison demonstrates that there is some consistency in how you would classify these targets based on $\mathrm{MAP}_{\mathrm{POD}}$ and the fragment docking protocol. However, the RSquare values are relatively low, 0.50 for SP and 0.55 for XP. Importantly, the lower scoring targets (PTP1B, ICE-1, IMPDH and CATK) are highlighted as difficult targets in both approaches. One most notable outlier between the two techniques is MDM2. This has been defined as a druggable target but it is clearly a challenging one due to the target binding site being a protein-protein interaction [24]. Aldose Reductase is an example in which the fragment docking protocol predicts the target to have a higher druggability than the Maximal Affinity Model.

The consistency of the ligandability assessment across different protein structures of the same target were also analyzed (Fig. 5). Where possible multiple protein structures were used to assess the ligandability of a target. The data shows that the choice of structure does impact on the ligandability score although in most cases it wouldn't significantly change your view of the target. The protein structures chosen for these assessments were chosen to maximize structural variation to give a more realistic view of the applicability of this method. However, 
it is clear that for some targets where a significant conformational change is required to form the binding site this approach will not successfully predict ligandability.

$<$ Figure 5>

To highlight the impact of the crystal structure chosen, for P38 and EGFR, an example of an active and inactive crystal structure was used. This was a DFG-in and DFG-out structure for P38 (1GF2 and 1KV2 respectively) and a c-Helix in and c-Helix out structure (1N1M and 1XKK respectively) of EGFR. In both cases the inactive structures scored slightly higher, this appears to be at least partly due to the fragments having a choice of two sub-pockets to choose from. One of these regions is at the 'hinge' region and the other is in the 'selectivity pocket' of the kinase [26]. The inactive structures also allow fragments to be buried in the deep lipophillic selectivity pocket which is often scored favorably. All scores quoted below are from the SP protocol which was shown to track well with XP for a given target. In P38, the inactive structure had a docking score of -6.6 whereas the score was -6 for the active conformation. In EGFR, the inactive structure had a score of -6.6 compared to the active structure with a score of -5.9 .

For the study of Enoyl Reductase [27], three crystal structures were used with four docking protocols. The structures 1C14 and 1ISZ both have cofactor and an inhibitor bound, the inhibitor binds to the protein-cofactor complex. In these systems the inhibitor was removed and docking carried out against the protein-cofactor complex. Fragment docking scores with the SP protocol of $-6.2(1 \mathrm{C} 14)$ and -6.5 (1ISZ) were observed for these two structures. An additional docking calculation was run on the $1 \mathrm{C} 14$ structure with the cofactor and inhibitor (Triclosan) removed to assess binding to the apo-enzyme, this gave a score of -6.1 . The final docking calculation was performed on the 1DFI structure which only has the cofactor bound. In this structure a loop in not visible which is observed to close over the active site when an inhibitor is bound (as in 1C14/1ISZ), resulting in a more open active site. When the docking protocol was carried out against 1DFI, with the cofactor present, you see a lower prediction (5.3) of ligandability, which shows that identifying the most suitable target structure can be important. The PTP1B example is also interesting, five of the protocols examining the primary binding site [28]. There is also a PTP1B structure of a ligand in an allosteric pocket [29] (1T4J) which was targeted in one run, this was the most highly scored of the structures by a small margin (-5.7 against a Mean score across the PTP1B structures of -5.2$)$. 


\section{Assessment of docking approach against target classifications}

The following assessment of the druggability of a number of targets has been suggested based on analysis of known inhibitors [8]. The key observation being the potency of the known inhibitors which is used to indicate the tractability of a target. Clearly for more novel targets, data on known inhibitors can be less abundant. The docking scores for the SP and XP protocols were compared against these defined categories of 'druggable', 'undruggable' and 'prodrug/transporter'. Again, the mean score of the fragments against the specified target was used to initially assess ligandability (Fig. 6).

\section{$<$ Figure 6>}

The docking protocol assessment demonstrated a significant separation between the 'druggable' and 'undruggable' classifications. Using the SP protocol the $\mathrm{p}$-Value $=0.0052$ and the XP protocol produced $\mathrm{p}$-Value $=0.0041$ when comparing the separation of these two categories by the ligandability score. However, the 'prodrug/transporter' examples spanned the mid-range of the two categories so were more difficult to differentiate. One outlier from the docking analysis was MDM2, defined as 'druggable' in the target classification. Although inhibitors are known for this target the binding site is challenging protein-protein interface so it is reasonable to expect this target to have a poorer ligandability assessment by docking. Although the 'prodrug/transporter' targets have had potent inhibitors successfully identified, it is expected that they will score less well by ligandability assessments using docking. The reasoning for this is that the active form of the inhibitor usually has a charged (or reactive) warhead which is masked in the prodrug to improve the properties of the compound [30]. As a number of highly charged or reactive functionalities will have been removed from fragment screening sets then tight binding fragments may not be identified. An alternative fragment set containing more highly charged/reactive functionalities may be required to further probe these targets. However, the fragment set used in this study remains a useful set to assess conventional ligandability and does contain a number of simple acids, bases and other charged groups.

The ligandability prediction from the mean score of all the docked fragments against a target was compared to the prediction using the mean score from just the top $10 \%$ ranked fragments against the target. The reasoning behind this being that some tractable targets may have a 
small number of tight binders which may not be reflected by the mean scores across the full fragment set. However this analysis did not show improved differentiation between the targets, the separation between the druggable and undruggable classes is actually slightly reduced $(p-$ Value $=0.0212$ for SP protocol and $p$-Value $=0.0944$ for XP). The results from the SP and XP docking protocols are shown in Fig. 7.

\section{$<$ Figure 7>}

An alternative way of expressing the ligandability data is to plot the docked scores of the ranked fragments of all the targets on a single plot. This gives a visual way of assessing if some targets have small numbers of fragments with high-predicted affinity or if the range of docking scores for a target has a flatter profile. It may be expected that a flatter profile shows a less interesting pocket than a profile which has a number of fragments predicted with a high affinity. The plot in Fig. 8 supports the early observation that COX-2 and Aldose Reductase are predicted to have a high ligandability from the docking studies. Thrombin and Acetylcholinesterase appear to have a number of highly scoring fragments; these targets are both classified as prodrug/transporter. This may reflect some differences in the difficulty of finding a lead (or fragment hit) compared to being able to modify that lead/fragment into an orally bioavailable drug. For example, it may be possible to identify hits against a certain target but the nature of the binding site may make it difficult to optimize the series whilst remaining in drug-like property space [31].

$<$ Figure 8>

To provide further information on how targets can be compared two additional scores were utilized. The 'Mean Score Target Adjusted' and the 'Mean Score Fragment Adjusted'. The Mean Score Targeted Adjusted is the ligandability score from the docking of the fragment set (using the mean score of the fragments) subtracted by the mean score of all the targets tested. So a negative number shows a target which is has a better predicted ligandability than the mean and vice versa. So clearly the rank ordering of the targets is not affected but it gives a benchmark for the comparison of new targets. As more targets are analyzed using this method then the mean score of all targets will change, especially in the initial stages, so this can be updated. The second score (Mean Score Fragment Adjusted) assesses how well each fragment scores to a single target compared to the mean score for that fragment across all targets. So 
this allows fragments to be identified with score highly against a certain target, but reduces the score for fragments, which score well in multiple targets. The intention being that the impact of the more lipophillic and featureless fragments, which may often act as frequent hitters, do not dominate the ligandability assessment. Therefore this score is compiled by subtracting the mean score of each fragment across all targets from the score against a specific target. The mean of this corrected score for fragments against a target is now used as a prediction of ligandability.

\author{
Mean Score Target Adjusted $=\quad$ ligandability score of target - mean \\ ligandability score of all targets
Mean Score Fragment Adjusted $=$ Mean (docking score of fragment against target - mean docking score of fragment against all targets)

These two scores are plotted in Fig. 9 for the assessed targets. SP and XP protocols are shown on the $\mathrm{x}$ - and $\mathrm{y}$-axis for both.

$<$ Figure 9>

The 'Mean Score Target Adjusted' allows a clearer comparison against how a studied target compares to the predicted average level of difficulty. Therefore allowing a benchmark against known targets which have previously been the subject of Lead Generation campaigns. The 'Mean Score Fragment Adjusted' doesn't significantly change the ranking ordering of most of the targets. This suggests that the affect of lipophillic frequent hitters in the assessment by docking is minimal as we aim to remove these from a fragment screening set. This way of scoring the targets could also be misleading if the number of examples from different target classes is not well balanced. This could lead compounds from over-represented target classes, such as kinases (CDK2, EGFR, P38 have been analyzed) to be artificially scored lower if similar fragments scored well between these targets. Two further methods of exploiting the docking scores were assessed. One method used a ligand efficiency score (docking score/heavy atoms) but this reduced the separation between the 'druggable' and 'undruggable' categories. Finally the most highly scored docked fragment was used to represent the 
ligandability of a target but this also failed to improve the separation between 'druggable' and 'undruggable' categories.

\section{Conclusions}

The docking method detailed provides an alternative method for assessing the druggability (or ligandability) of target proteins where crystal structures are available. When using the mean docking score for the fragment set against a target the results are largely consistent with the experimental data and approaches reported previously in the literature. Further validation work is to be performed on in-house targets which have already been targeted by lead generation approach in addition to novel enzymes currently under target selection. Where there are a number of targets which could be prosecuted, of a similar strength of disease linkage, then this information may be able to prioritize targets most likely to return lead-like compounds. This approach may also help to identify the most tractable pocket of a target and may guide the Lead Generation approach (i.e. Virtual/Directed Screen, HTS, FBLG or a combination of these). Other information will be used in this decision making process, including the knowledge of known ligands/substrates/co-factors of the target and any relevant information or experience around the target class. Clearly the limitation of this approach is the requirement for crystal structures to be are available, or where there may be a structure of a close homologue. There is also an additional issue around protein flexibility which suggests that examples which have an allosteric pocket or a significant degree of conformational change in the active site will not be accurately predicted. In addition, for some targets, the primary binding site may not be clearly located so a tool like SiteMap may be required to rank potential binding sites before they are subsequently analyzed. Often, once all these factors and additional information are considered, a high throughput measure to assess large numbers of targets may not be required. However, this approach has shown that a range of targets can be analyzed using this methodology and the more chemically tractable examples can be prioritized in many cases. This approach is to be further evaluated using fragment sets with a broader range of physicochemical properties, this may be particularly valuable for proteinprotein interfaces. For druggability assessment using these fragments, computational approaches may have additional value due to the potential issues around the experimental screening of more lipophillic fragments with poor solubility. Another extension of this 
approach may the integration of additional methods which may consider protein flexibility. Further work around the binding mode and subsequent affinity predictions of fragments will also be required to increase the predictive power of this methodology.

\section{Acknowledgments}

I would like to acknowledge the significant background work from a number of colleagues across AstraZeneca, which is described in this study. Additional thanks to Alex Breeze for introducing the term 'ligandability'. 


\section{References}

1. Brown D, Superti-Furga G (2003) Rediscovering the sweet spot in drug discovery. Drug Discovery Today 8:1067-1077

2. Hajduk PJ, Huth JR, Fesik SW (2005) Druggability indices for protein targets derived from NMR-based screening data. J Med Chem 48:2518-2525

3. Hopkins AL, Groom CR (2002) The druggable genome. Nat Rev Drug Disc 1:727-730

4. Hajduk PJ, Huth JR, Tse C (2005) Predicting protein druggability. Drug Discovery Today $10: 23-24: 1675-1682$

5. Burgoyne NJ, Jackson RM (2006) Predicting protein interaction sites: binding hot-spots in protein-protein and protein-ligand interfaces. Bioinformatics 22:1335-1342

6. Fuller JC, Burgoyne NJ, and Jackson RM (2009) Predicting druggable binding sites at the protein-protein interface. Drug Discovery Today 14:3-4:155-161

7. Cheng AZ, Coleman RG, Smyth KT, Cao Q, Soulard P, Caffrey DR, Salzberg AZ, Huang ES (2007) Structure-based maximal affinity model predicts small-molecule druggability. Nat Biotechnol 25:71-75

8. Jhoti H (2001) High-throughput structural proteomics using x-rays. Trends Biotechnol 19:67-71

9. Koshland DE (1958) Application of a Theory of Enzyme Specificity to Protein Synthesis. Proc Natl Acad Sci 44:98-104

10. Whitty A, Kumaravel G (2006) Between a rock and a hard place? Nat Chem Biol 2:112118

11. Albert JS, Blomberg N, Breeze AL, Brown AJH, Burrows JN, Edwards PD, Folmer RHA, Geschwindner S, Griffen EJ, Kenny PW, Nowak T, Olsson LL, Sanganee H, Shapiro AB (2007) An Integrated Approach to Fragment-Based Lead Generation:Philosophy, Strategy and Case Studies from AstraZeneca's Drug Discovery Programmes. Curr Top Med Chem 7:1600-1629

12. Congreve M, Carr R, Murray C, Jhoti HA (2008) 'Rule of Three' for fragment-based lead discovery? Drug Discovery Today 8:Issue 19:876-877

13. Kim R, Skolnick J (2008) Assessment of programs for ligand binding affinity prediction. J Comput Chem 8:1316-1331

14. Lipinski CA (1997) Adv Drug Del Rev 23:3-25

15. Berman HM, Westbrook J, Feng Z, Gilliland G, Bhat TN, Weissig H, Shindyalov IN, Bourne PE (2000) The Protein Data Bank. Nucleic Acids Res 28:235-242 
16. Maestro; Schrödinger: San Diego, CA 92122-1003

17. Hooft RWW, Sander C, Vriend G (1996) Proteins 26:363-376

18. Halgren T (2007) New Method for Fast and Accurate Binding-site Identification and Analysis. Chem Biol Drug Des 69:2:146-148

19. Lyne PD, Kenny PW, Cosgrove DA, Deng C, Zabludoff S, Wendoloski JJ, Ashwell S (2004) Identification of Compounds with Nanomolar Binding Affinity for Checkpoint Kinase-1 Using Knowledge-Based Virtual Screening. J Med Chem 47:1962-1968

20. CORINA; Gasteiger Research; Erlangen, Germany

21. Halgren T (1996) A Merck Molecular Force Field: I-V. J Comput Chem 17:490-641

22. Grant JA, Pickup BT, Sykes MJ, Kitchen CA, Nicholls A (2007) Chem Phys Lett 441: 163-166

23. GLIDE (Version 50207); Schrödinger: San Diego, CA 92122-1003

24. Vassilev LT, Vu BT, Graves B, Carvajal D, Podlaski F, Filipovic Z, Kong N, Kammlott U, Lukacs C, Klein C, Fotouhi N, Liu EA (2004) In vivo activation of the p53 pathway by small-molecule antagonists of MDM2. Science 6:844-848

25. JMP, Version 6. SAS Institute Inc., Cary, NC, 1989-2007

26. Buchanan SG (2003) Protein structure: discovering selective protein kinase inhibitors. Targets 2:101-108

27. Roujeinikova A, Levy CW, Rowsell S, Sedelnikova S, Baker PJ, Minshull CA, Mistry A, Colls JG, Camble R, Stuitje AR, Slabas AR, Rafferty JB, Pauptit RA, Viner R, Rice DW (1999) Crystallographic analysis of triclosan bound to enoyl reductase. J Mol Biol 294:527-535

28. Zhang S, Zhang ZY (2007) PTP1B as a drug target: recent developments in PTP1B inhibitor discovery. Drug Discov Today 12:373-81

29. Wiesmann C, Barr KJ, Kung J, Zhu J, Shen W, Fahr BJ, Zhong M, Taylor L, Randal M, McDowell RS, Hansen SK (2004) Allosteric inhibition of protein tyrosine phosphatase 1B. Nat Struct Mol Biol 11:730-737

30. Rautio J, Kumpulainen H, Heimbach T, Oliyai R, Oh D, Järvinen T, Savolainen J (2008) Prodrugs: design and clinical applications. Nat Rev Drug Discovery 7:255-270

31. Leeson PD, Springthorpe B (2007) The influence of drug-like concepts on decisionmaking in medicinal chemistry. Nat Rev Drug Discovery 6:881-890 


\section{Tables}

Table 1 X-ray crystal structures used in ligandability assessment by docking

\begin{tabular}{|c|c|}
\hline Protein Name & PDB Codes \\
\hline Enoyl Reductase & 1C14, 1DFI, 1ISZ \\
\hline Aldose Reductase & 1PWL, 1T41, 1PWM \\
\hline CDK2 & 1E1X, 1H07, 1H08, 1HCK, 1KE6, 1KE8, 1KE9 \\
\hline Cyclo-oxygenase 2 & 4COX \\
\hline EGFR & 1N1M, 1XKK \\
\hline MDM2 & 1KV2, 2GFS \\
\hline P38 & 1O86 \\
\hline Acetylcholinesterase & 1NF7 \\
\hline IMPDH & 1MEM, 1NLJ \\
\hline Thrombin & 1BMQ \\
\hline CATK & 1GFI, 1NNY, 1ONZ, 1PTY, 1Q1M, 1T4J \\
\hline ICE-1 & \\
\hline PTP1B &
\end{tabular}




\section{Figure captions}

Fig. 1 Property profile of fragment set for 'ligandability' studies using docking

Fig. 2 Comparison of SP and XP protocol mean docking scores across targets against ClogP and Molecular Weight. 2A and 2B show the Glide SP results with 2C and 2D showing the XP results

Fig. 3 Comparison of Ligandability prediction from SP and XP Glide docking. SP score (x-axis) against XP score (y-axis)

Fig. 4 Comparison of the fragment docking approach with the Maximal Affinity Model (expressed in $\mathrm{pKd})$. The SP protocol data is shown in 4A (RSquare=0.50), the XP data is in plot $4 \mathrm{~B}(\mathrm{RSquare}=0.55)$

Fig. 5 One-way Anova Analysis [25] of the fragment docking scores from different protein structures of a target. The SP protocol data is shown in Fig. 5A and the XP data in Fig. 5B

Fig. 6 Comparison of docking scores against target classification using potency of known inhibitors [8]. SP protocol shown in Fig. 6A and XP protocol in Fig. 6B

Fig. 7 Comparison of ligandability scores by docking against chemical tractability guided by knowledge of known inhibitors [8]. The mean docking score of the top $10 \%$ of fragments against a target defined ligandability in this study. SP protocol shown in Fig. 7A and XP protocol in Fig. 7B

Fig. 8 Profile of the docked fragment scores (SP protocol) for each target in the validation set

Fig. 9 Comparison of adjusted docking scores to assess ligandability. Fig. 9A shows the 'Mean Score Target Adjusted'. Fig. 9B shows the 'Mean Score Fragment Adjusted' 


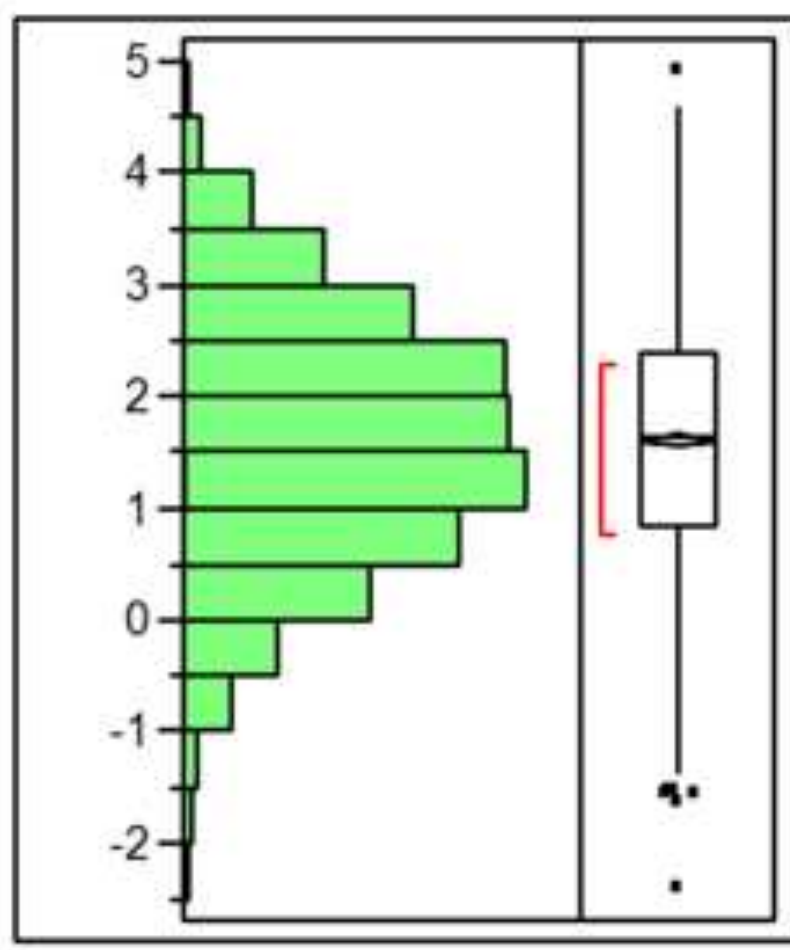

ClogP

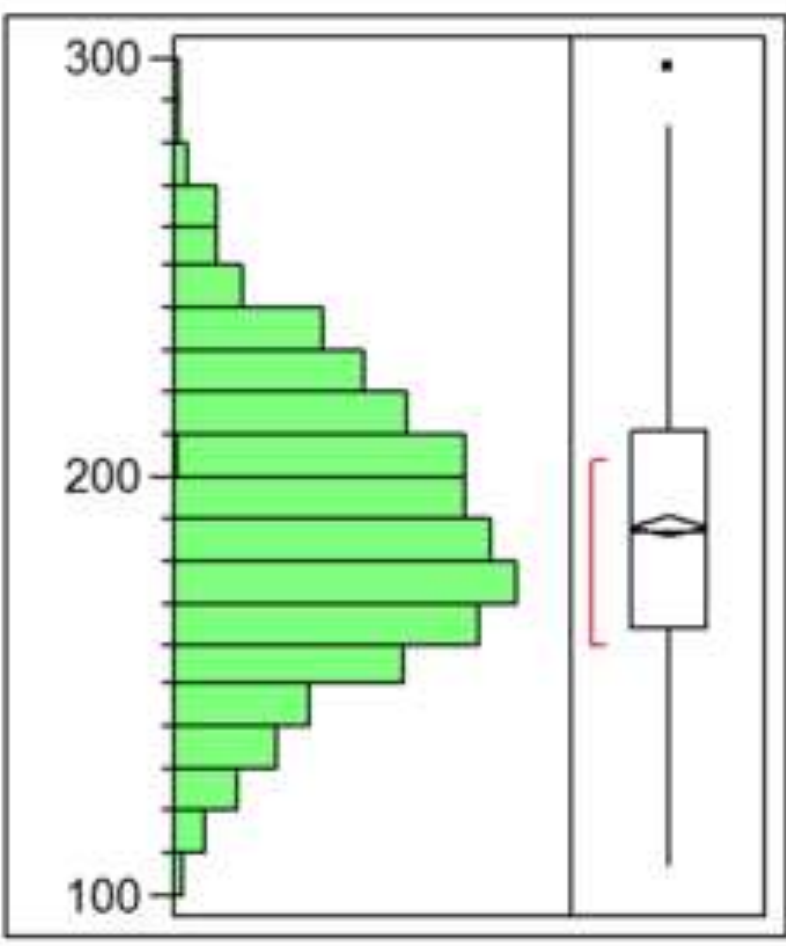

Molecular Weight

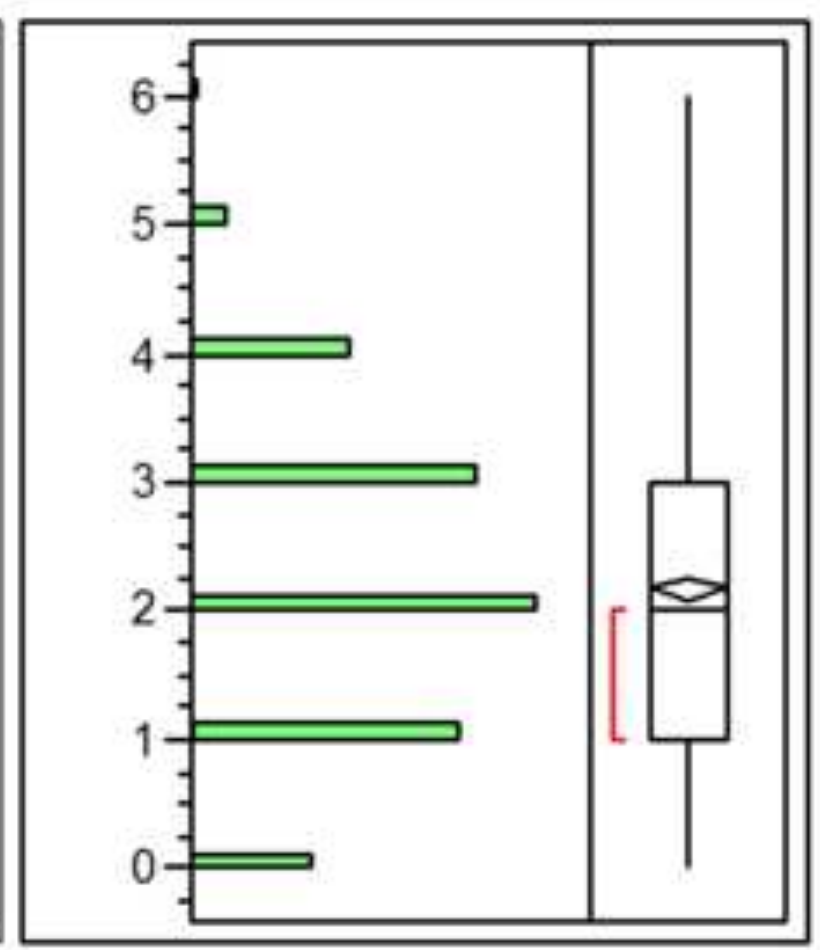

Rotatable Bonds

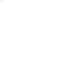


Click here to download high resolution image
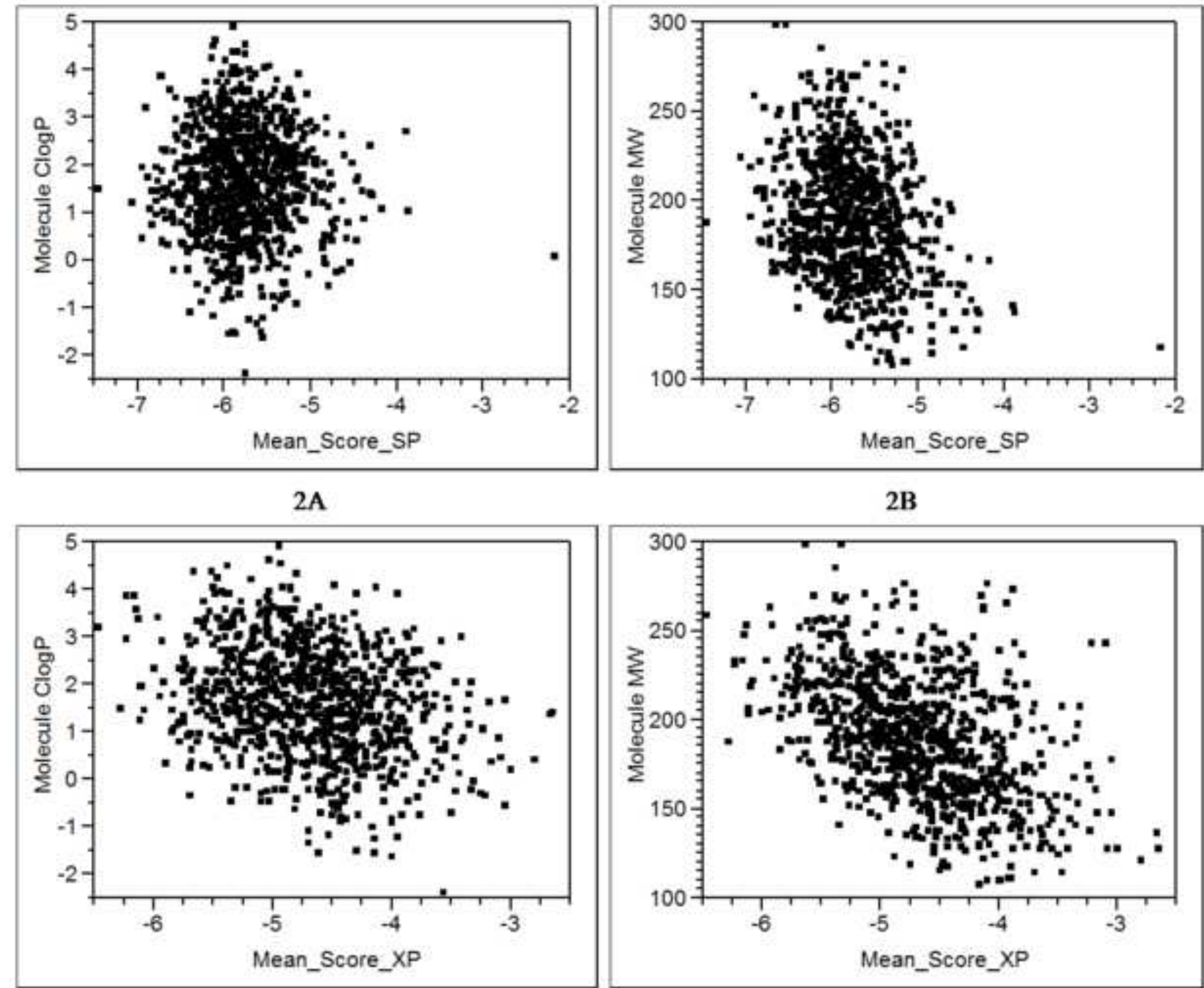

2C

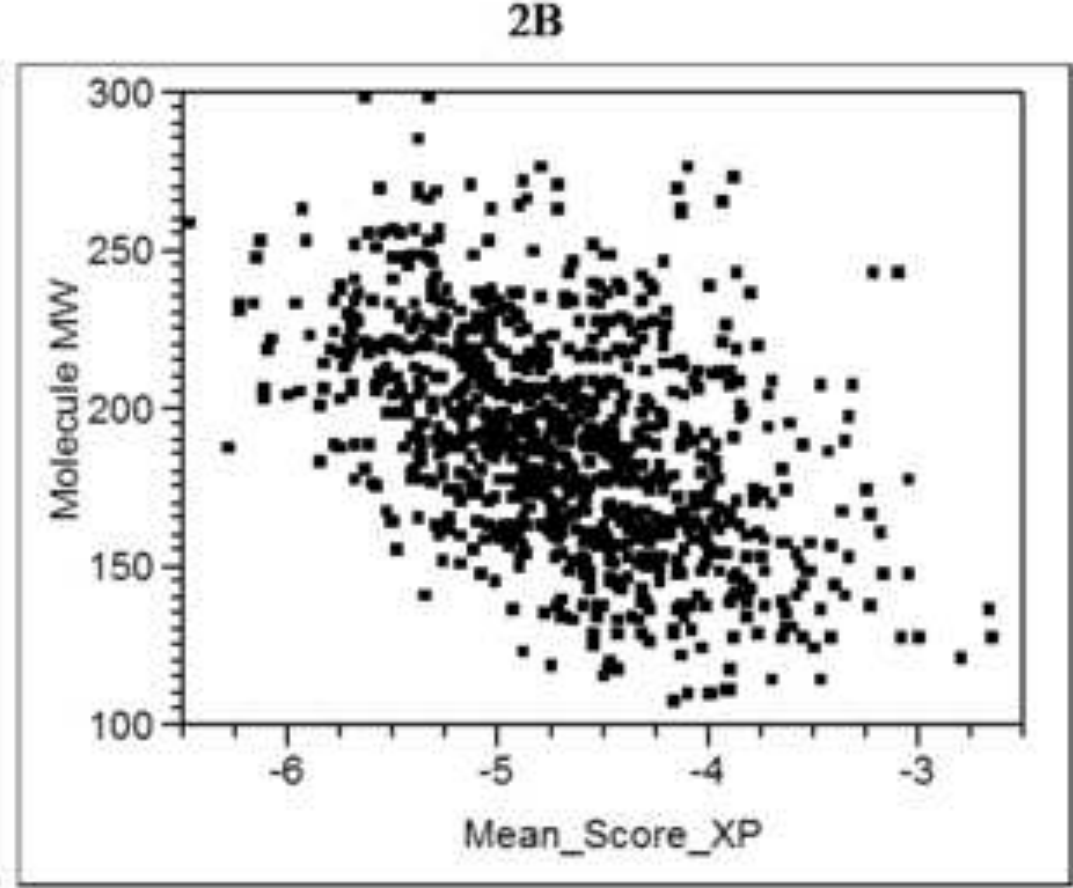

2D 


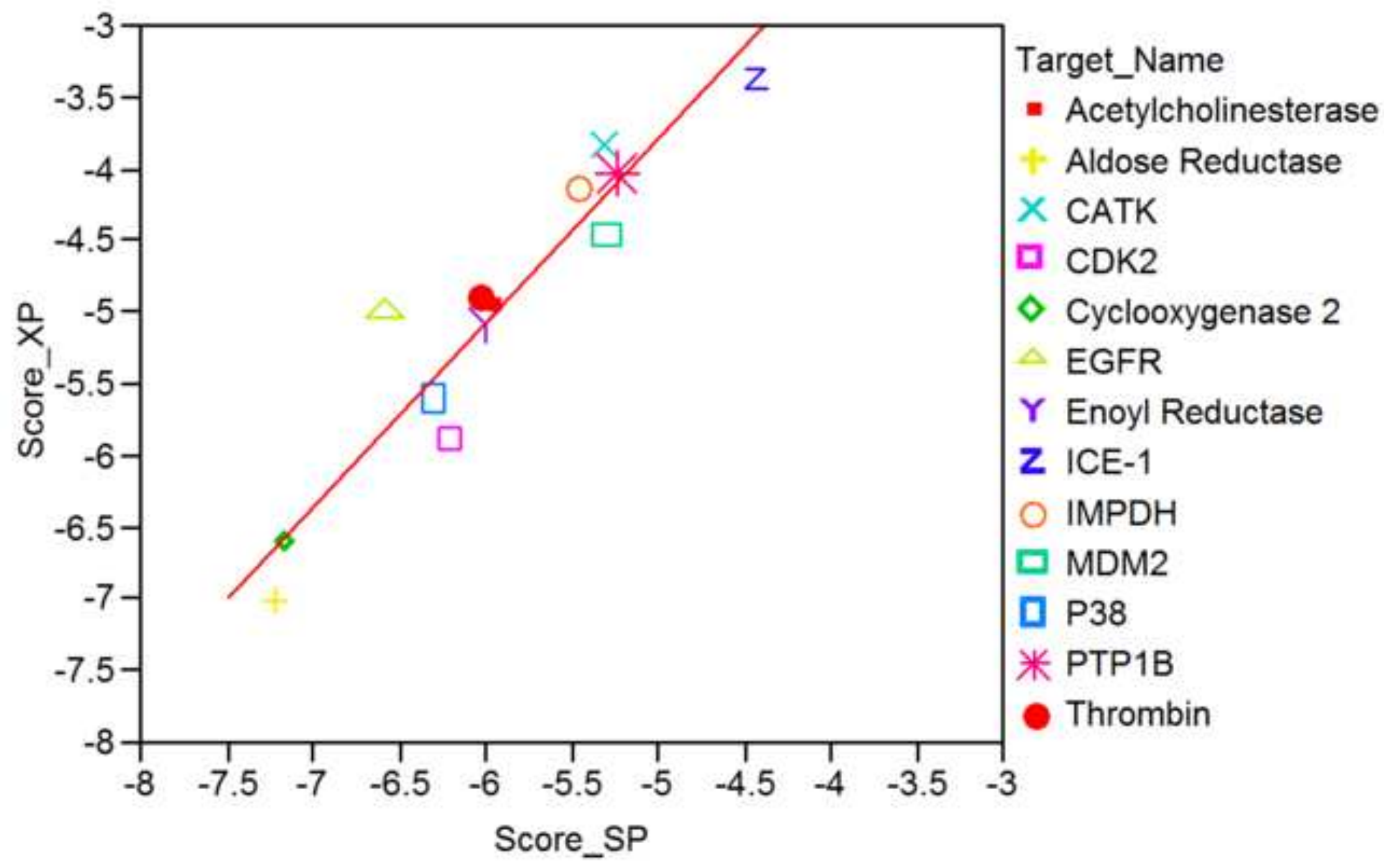


Click here to download high resolution image
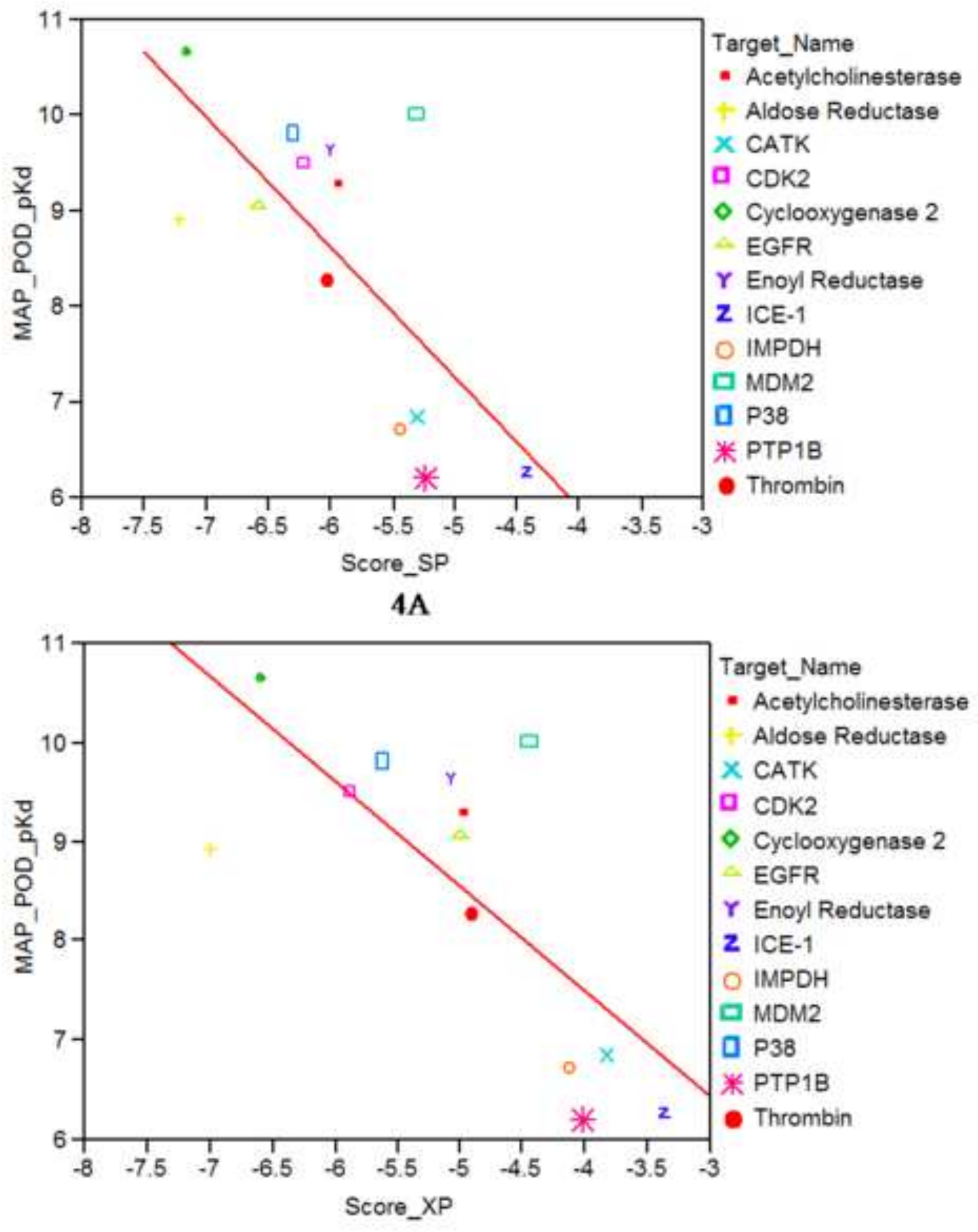

4B 
Figure 5

Click here to download high resolution image

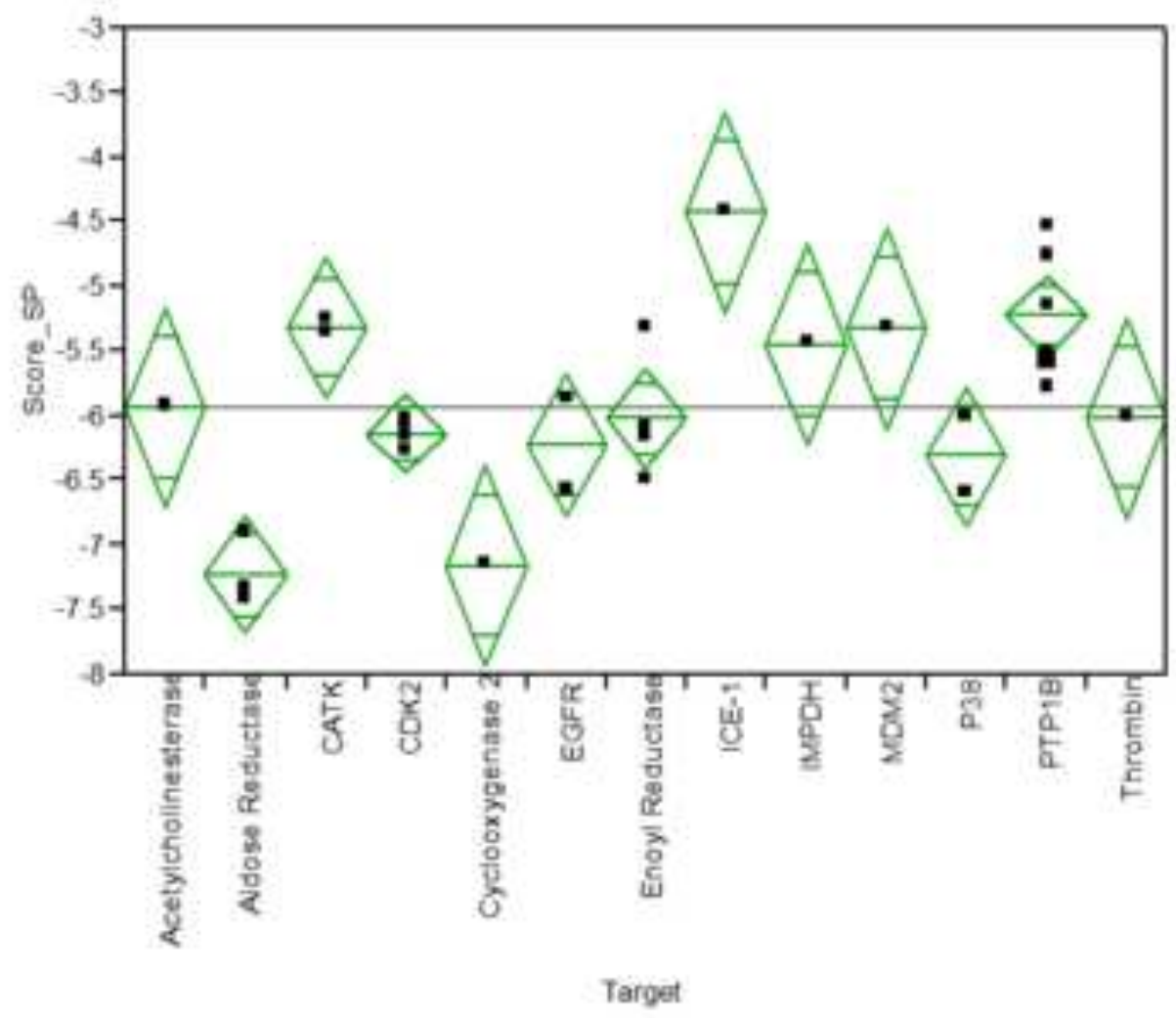

$5 A$

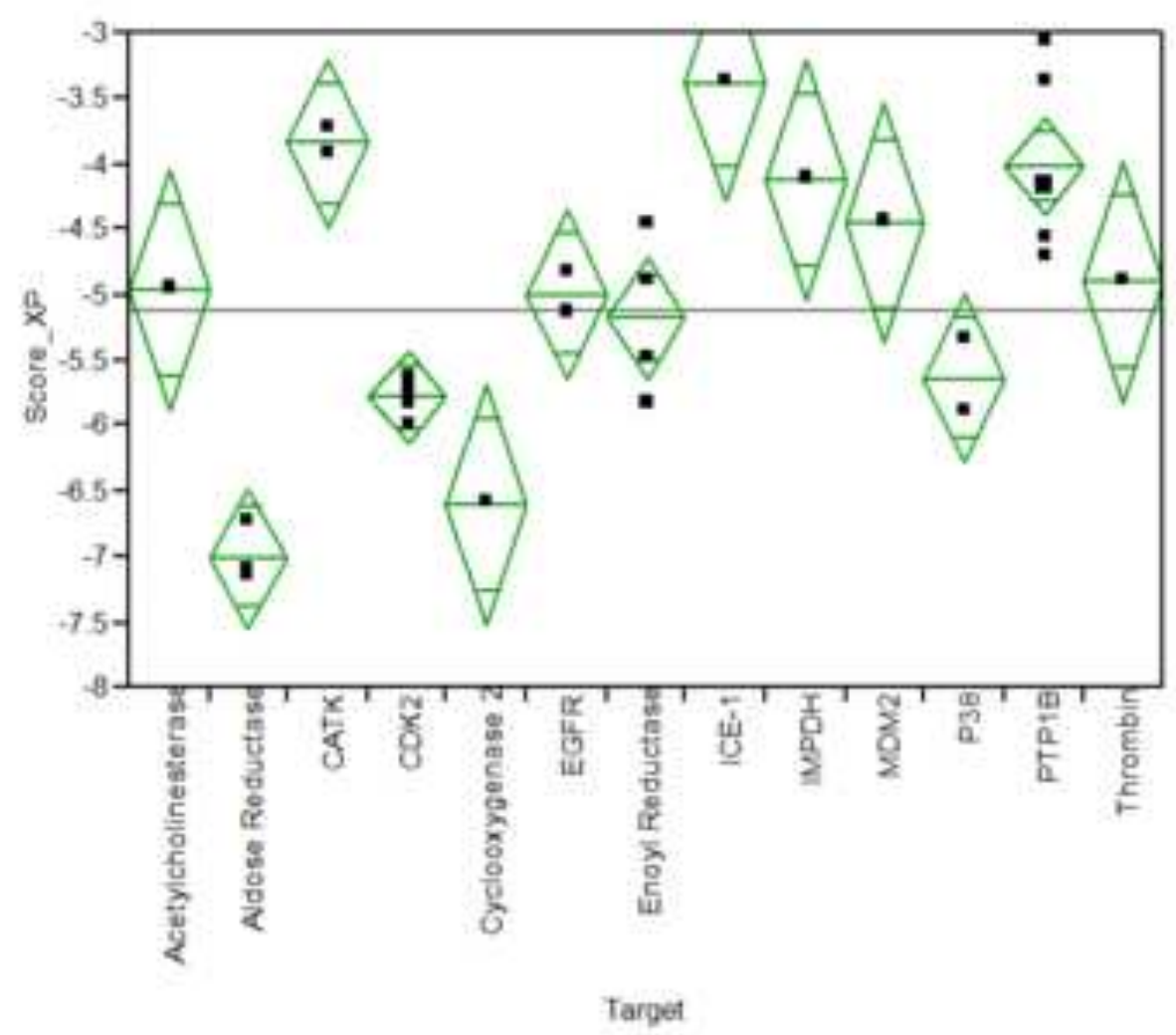


Click here to download high resolution image
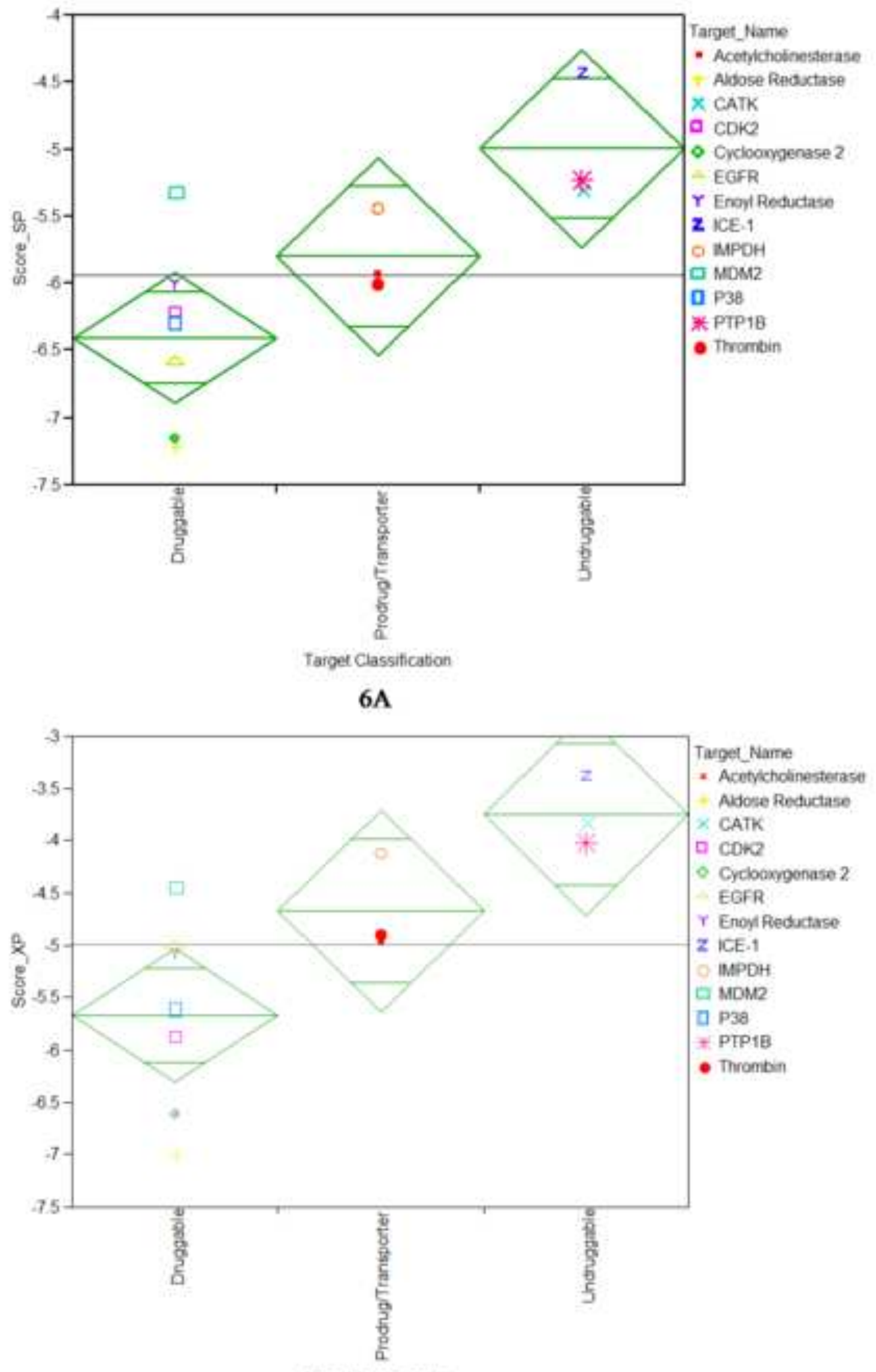

Tarpot Cursaification

$6 \mathrm{~B}$ 


\section{Figure 7}

Click here to download high resolution image

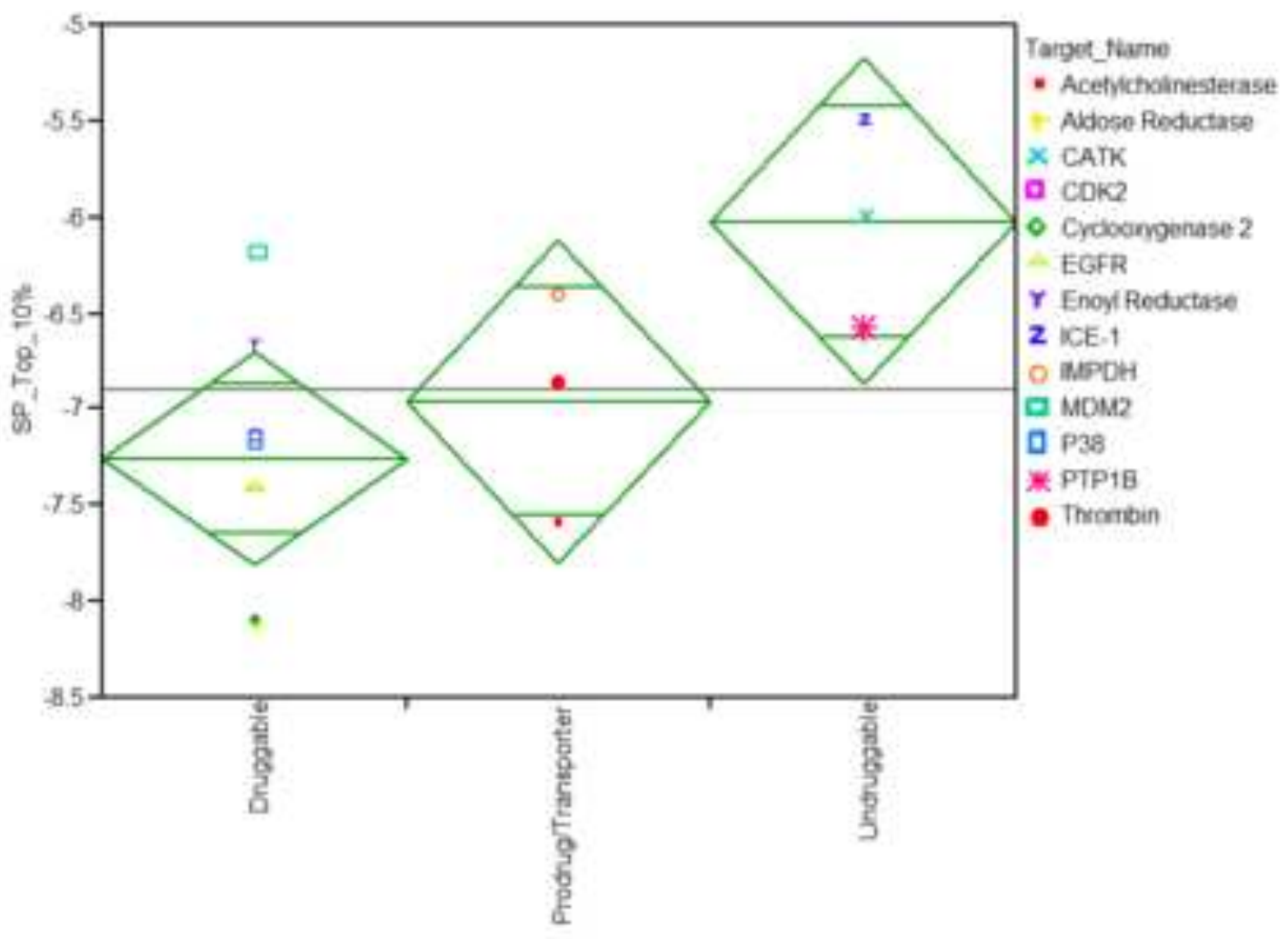

Target Classification

$7 \mathrm{~A}$

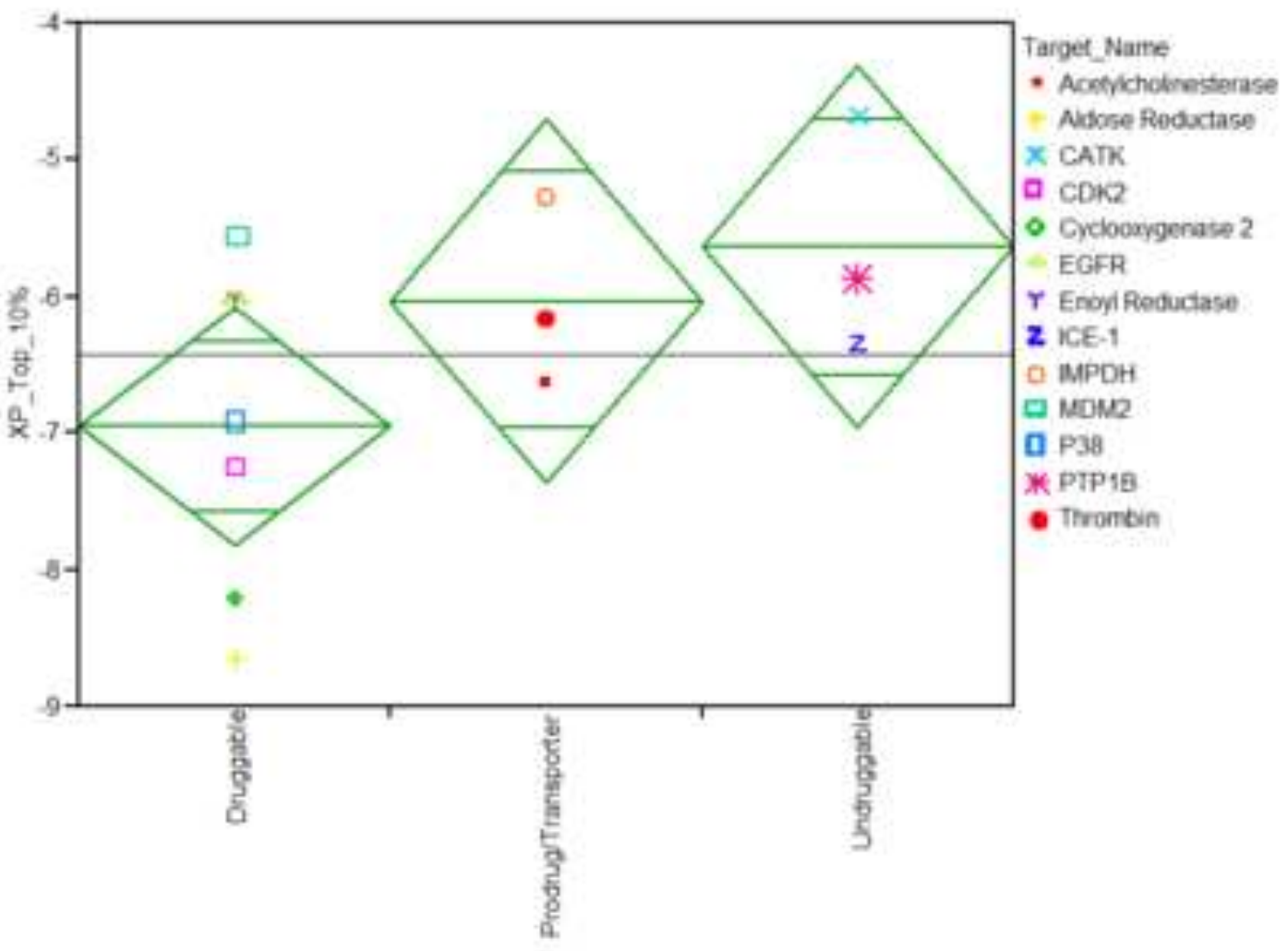

Target Classification 
Click here to download high resolution image

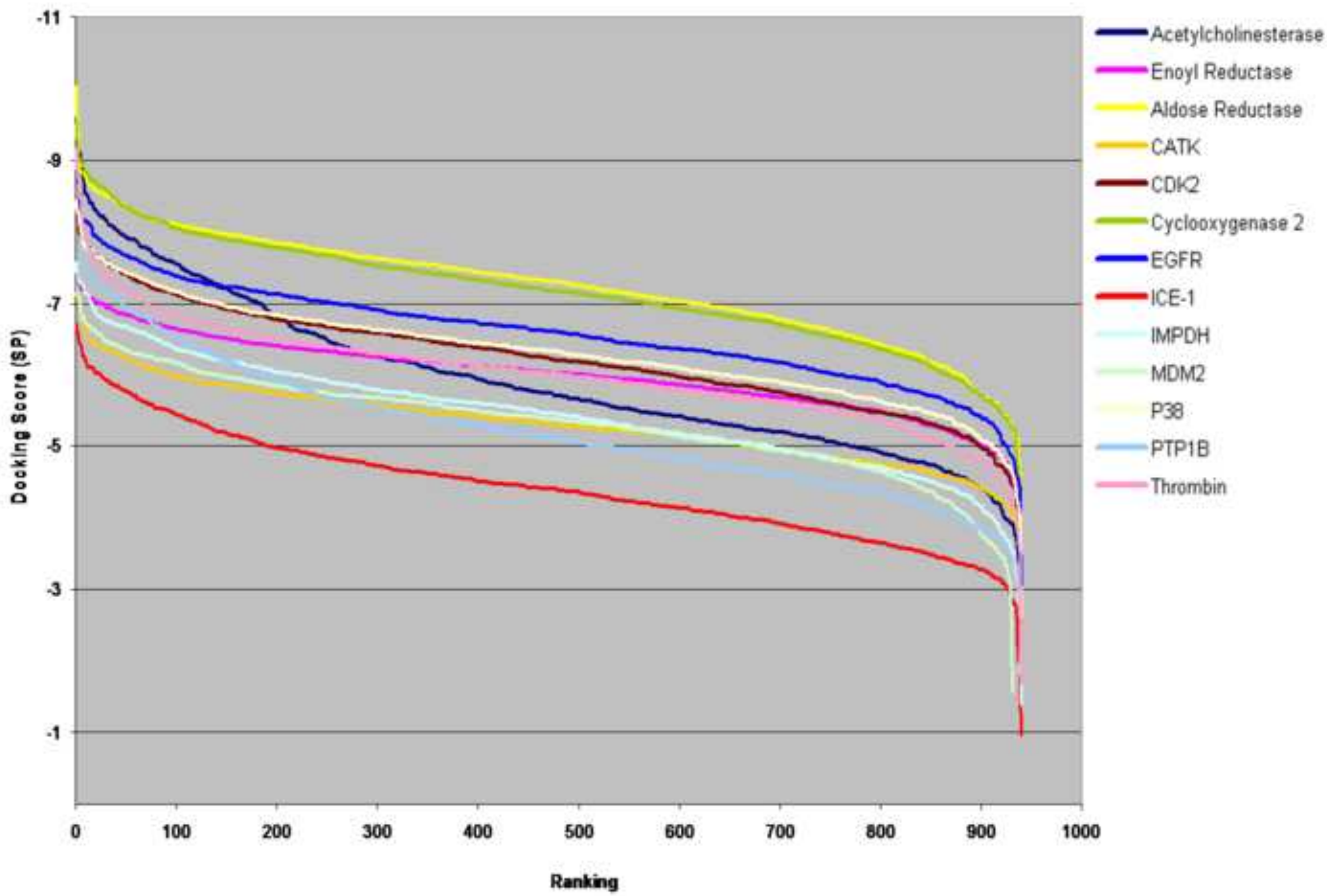


Figure 9
Click here to download high resolution image

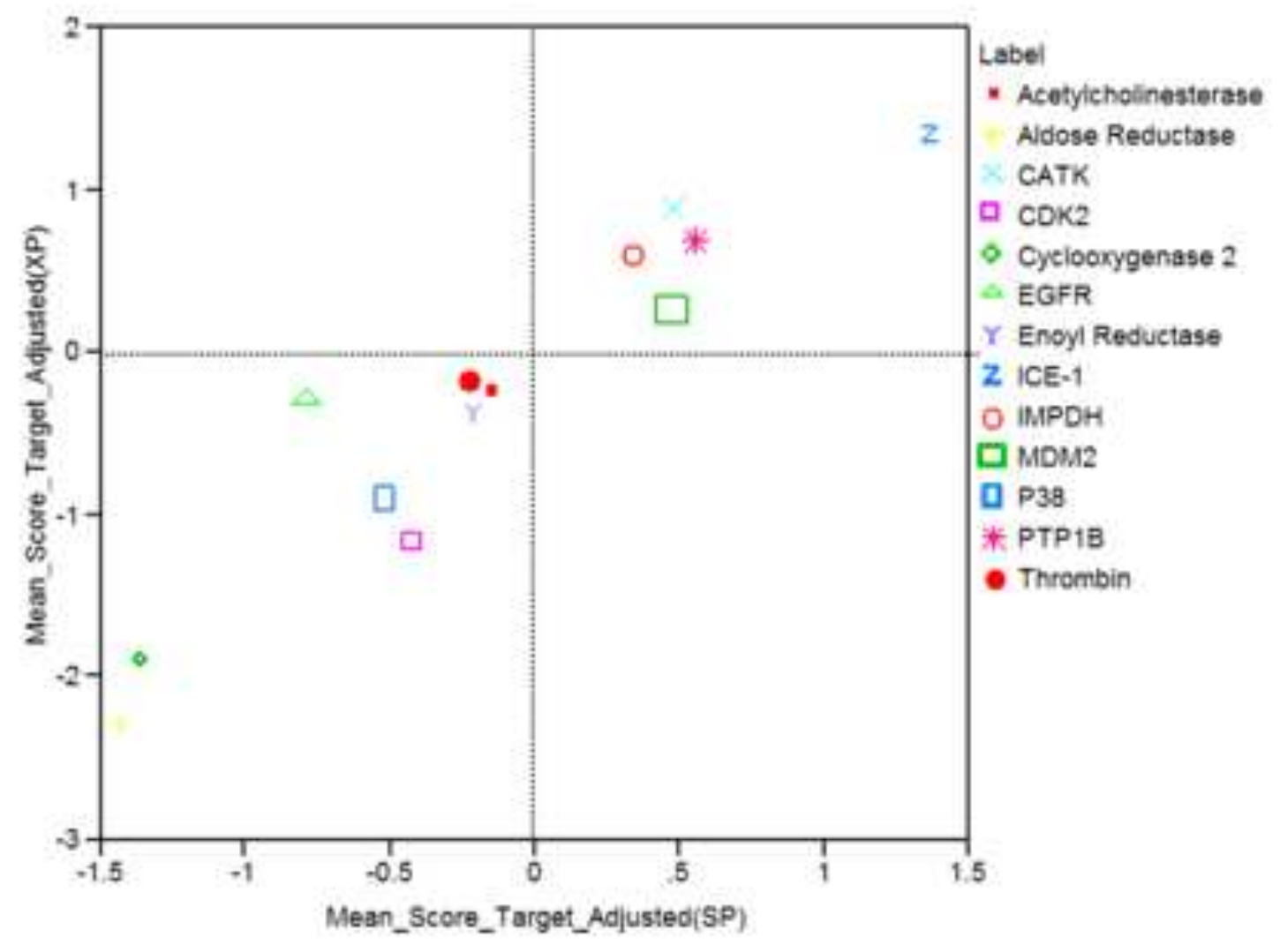

9A

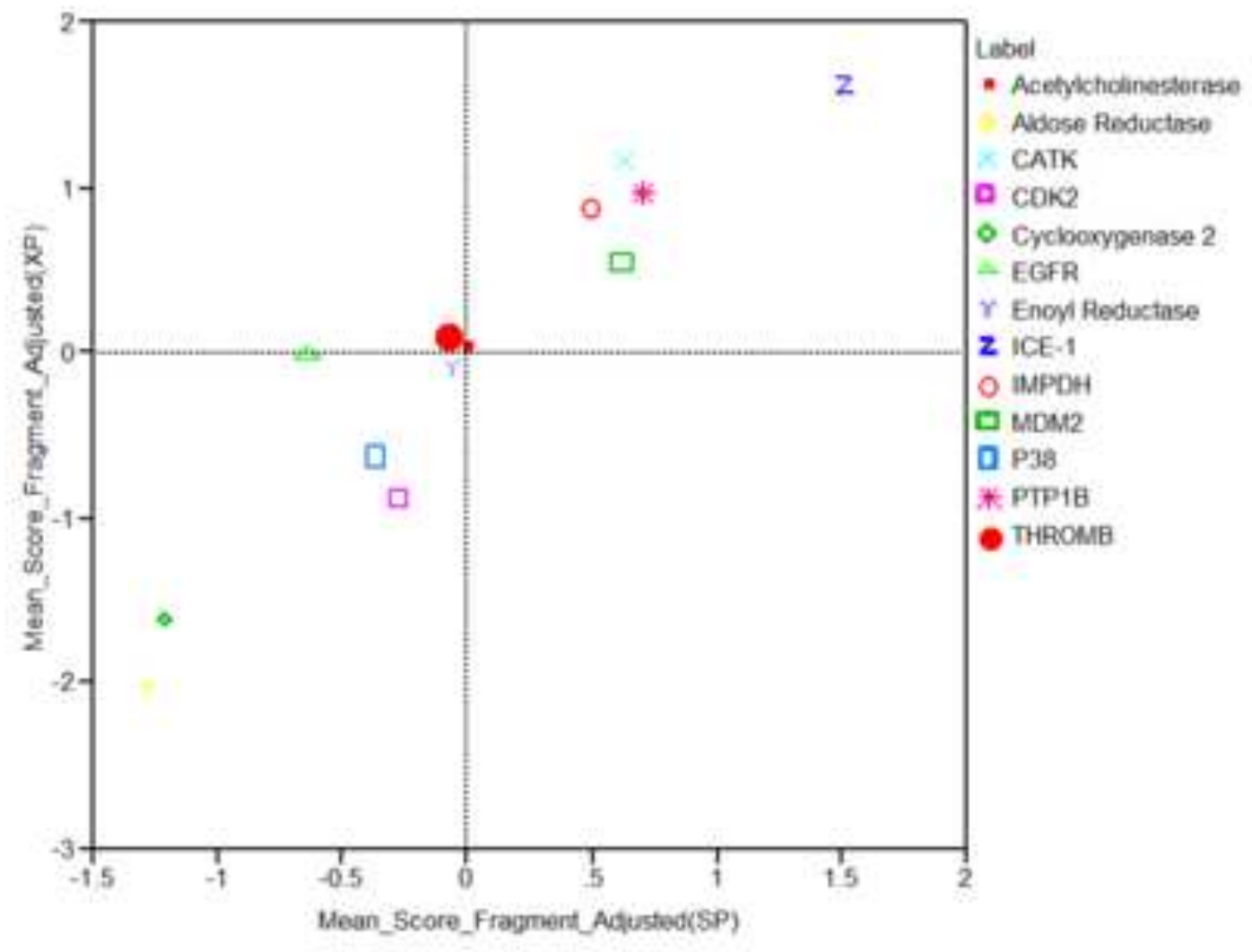

